\title{
Chloroplast Genome of Vigna aconitifolia and Localization of Certain Photosynthesis-related Genes
}

N Y Kelkar', A K Tyagi' and S C Maheshwari', 2

'Department of Botany, University of Delhi, New Delhi - 110007

2Department of Plant Molecular Biology, University of Delhi, South Campus, New Delhi-110 021, India

Due to an oversight, an incorrect version of Fig. 3 has been printed in the above article (J. Plant Biochem. Biotech 1: 1-4, 1992). The correct version along with the legend is given below.

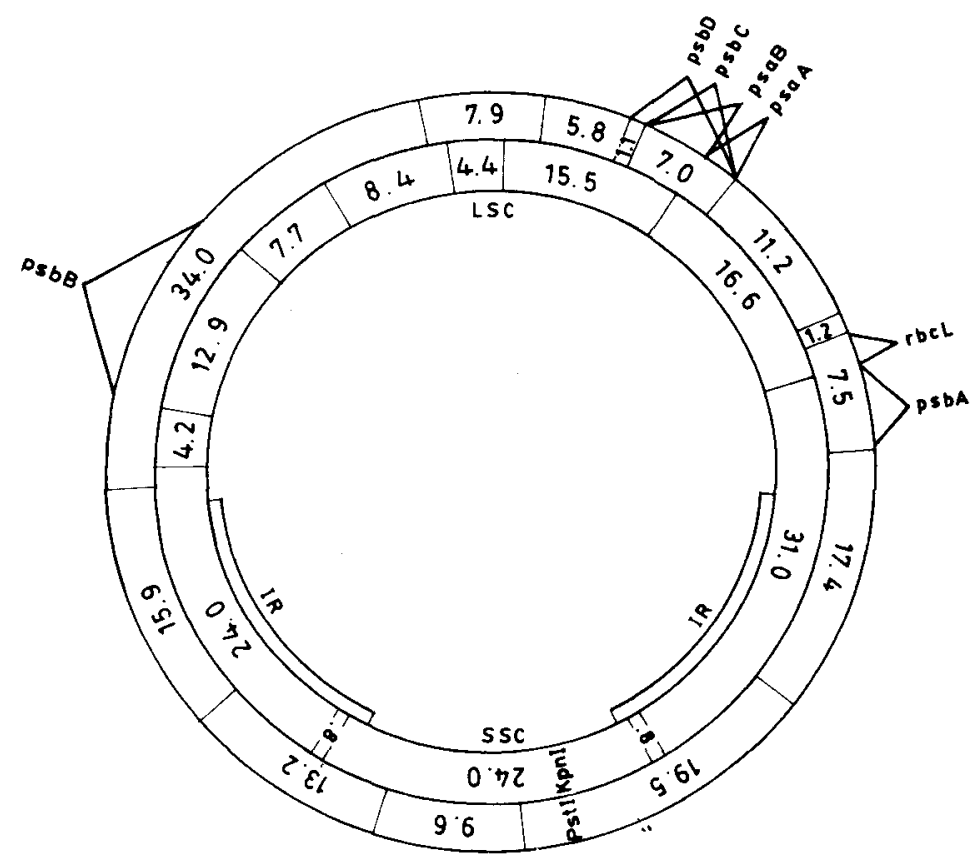

Fig. 3. The position of various genes on the Pst and Kpnl generated physical map of $V$. aconitifolia which is based in part also on the information concerning $V$. radiata cpDNA (8). The sizes $(\mathrm{kb})$ of various fragments generated by $P s t$ and $K p n l$ are also given. Abbreviations: IR, inverted repeats; LSC, large single copy region; SSC, small single copy region. 